\title{
Mathematical model of ageing of natural and manmade objects in monitoring systems
}

\author{
(C) V. Mostovyy, S. Mostovyi, 2010 \\ Institute of Geophysics, National Academy of Sciences of Ukraine, Kiev, Ukraine \\ mostovoy1@voliacable.com
}

The phenomenological mathematical model of an estimation of fatigue and aging processes of natural and manmade objects is proposed in this work. The model is based on the mainstream representations existing in material science. Information, obtained by authors while monitoring the Fourth Block of Chernobyl Nuclear Power Plant and the industrial constructions, allows to state, that the process of aging can be represented in space of attributes, each component of which describing an elastic property of materials, which form an investigated object. Since speeds of propagation and the form of pressure and share waves depend on the elastic properties of the materials, it follows that the changes of these properties results in the changes of spectral characteristics of the emission signals. One can see such changes in aging materials. Thus, while monitoring the Forth Block of Chernobyl Nuclear Power Plant, we observed that the Fourier spectrum of a background of natural emission have shifted from the one, that had been stationary before.

More specifically the center (meidan) of the spectrum became lighter and the tails (low- and highfrequency areas) became heavier. This effect can be explained by two major reasons. The first reason is the defragmentation of the elements of the construction, that results in an increase of eigenfrequencies of separate fragments due to a decrease of their geometric sizes. The second reason is an increase of geometric sizes of the defects (fractures) of separate fragments of the object, that results in a decrease of the frequency of emission signals of these defects.

Later the authors applied a similar approach to investigation of the objects that age less intensively. The eigenfrequencies of such objects were in the seismic spectrum band. In a stationary state in the regime of passive monitoring we observed that the vector, describing the state of the object, was migrating within a rather small ellipsoid of rotation in the space of attributes. Such behavior was observed until an occurrence of fatigue. From the point of view of materiology, fatigue is the developing local structural damages arising under the cyclic loadings of a material. Typically, the maximal values of the stress in a cycle are smaller than the highest stress limit for a given material.

Structural failure of a material consists in loss of bearing ability of an element or the whole structure. It begins when tensions in the material become close to limiting ones, causing excessive deformations, and when the material during a full cycle does not come back to an initial state, displaying the phenomenon of hysteresis.

Any redistribution of energy in a material is accompanied by the origination of the emitted signals. Emission of signals is the stress waves generated by a sudden internal redistribution of stress in a material. In turn such a redistribution is typically caused by sudden changes in the internal structure of a material. The possible reasons of the internal structure changes are occurrence and growth of fractures, movement and transformation of a phase in monolithic materials, and disintegration of its components.

Cyclic tensions result in fatigue of a material. Fatigue results in the irreversible deformations. In the model that we consider the process of aging is defined by changes of parameters of a material. It is represented in a space of attributes.

In particular, such structural changes in time stem from the geological faults, which are the basic sources of earthquakes.

If one models behavior of geological faults as behavior of the materials, then one can consider the signals of emission generated by a fault to be a source of information about their fatigue and aging. 
Dynamics of the parameters of the emission signals reflects changes of elastic properties of a substance in faults. It means that the changes of the dynamic parameters of the emission signals are connected with the dynamic characteristics of these faults. Moreover, due to presence of a stochastic background noise at monitoring procedure, one needs to have a model that takes such randomness into account.

The key question is the choice of informative parameters for the space of attributes, in which it is necessary to carry out the dynamic analysis of behavior of the parameters, and to build a solving rule for a forecast of a fault condition based on this analysis.

Thus the choice of the space of attributes that represents aging and fatigue becomes a crucial problem. Since information about the condition of an object is in the form of characteristics of emitted signals, and consequently information is indirect; we can only carry out the indirect measurements of propagation of emission waves. The dynamic changes of these waves are represented in the dynamics of their spectral characteristics. The components of the space of spectral characteristics represent the parameters, which are statistically connected to the characteristics of an object.

Our mathematical model takes into account two stochastic components of emission process. The first one is the point process of the moments of occurrence of emitting signals; the second one is the process of formation of a shape of the fluctuating signals. Stochastic characteristics of such a compound random process reflect the change of the elastic characteristics of the material.

The problem of estimation of the parameters of the considered stochastic process is reduced to an estimation of their a posteriori distribution. The dynamics of these parameters represents the processes of accumulation of fatigue and aging of the object. Our decision-making model for evaluation of the level of fatigue is based on risk criteria.

The space of attributes is a subspace of the image space of wavelet transform. In this space the emissions signals are represented by the amplitudes, scale factors and parameters of shift.

In the model it is possible to use both physically feasible signals, i.e. signals that satisfy the conditions of causality and stability, and physically unfeasible signals, i.e. the signals that do not satisfy these conditions. To represent physically feasible functions one can use the orthonormal system of the Haar functions in a Hilbert space, where inner product is given by an integral of the product of the functions, and the norm of a function is the standard $L^{2}$ norm. To represent emission signals occasionally it is convenient to choose physically unfeasible functions, which satisfy the condition of stability, but do not satisfy the condition of causality.

For example, consider the derivatives of the density function of the normal distribution with free parameters ó and ì. The obtained functions are not orthogonal (in the Hilbert space defined above), but, if the the parameters ó and ì take values in a certain set, these functions can be close to orthogonal, i.e. the scalar product of two functions with various ó and ì can be close to zero.

Now, consider passive monitoring. Information about an object is enclosed in emission signals, which are registered together with a natural background noise. It is natural to assume, that these signals form a stochastic process. One can model such a process in many different ways, e.g. as a Poisson process, which is described by only one parameter, or as a process, which is described by a vector of parameters of a large dimension. On the one hand the latter model is more complicated and it requires more a priori information about an object, which is being monitored. On the other hand it can give more insight about it and thus is useful. An example of such a process is a non-stationary binomial (or Bernoulli) process.

In order to take into account a natural background noise, one can model it as a stochastic process, which additively accompanies the process of registration of the emission signals. It can be assumed to be a stationary or a quasi-stationary stochastic process. Quasi-stationarity might stem from a seasonal or a daily change of the parameters of the underlying process.

The usage of a wavelet transform for an approximation of emission signals allows one to minimize the number of parameters of an approximation of a signal, since the wavelet basis can be formed from a fragment of the (random) process of emission of signals.

Under an investigation of a real object the system that we built collects data, processes it in real time and makes a decision about the state of the object. It important to emphasize that our system is a self-learning system, thus it uses data to learn the stationary state and the dynamics of the changes of the object. 